\title{
Hsa-mir-548 family expression in human reproductive tissues
}

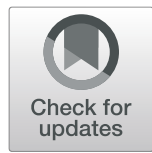

\author{
IImatar Rooda ${ }^{1,2^{*}}$, Birgitta Kaselt ${ }^{1}$, Maria Liivrand ${ }^{1}$, Olli-Pekka Smolander ${ }^{1}$, Andres Salumets ${ }^{2,3,4,5}$ and \\ Agne Velthut-Meikas ${ }^{1}$
}

\begin{abstract}
Background: Hsa-miR-548ba expressed in ovarian granulosa cells targets PTEN and LIFR, which are essential for ovarian follicle activation and growth. The expression pattern of hsa-miR-548ba correlates with its host gene folliclestimulating hormone receptor (FSHR), and FSH has a positive influence on hsa-miR-548ba expression. However, hsamiR-548ba is a member of a large hsa-mir-548 family with potentially overlapping targets. The current study aims to investigate the co-expression of hsa-mir-548 family members in FSHR-positive reproductive tissues and to explore the potential co-regulation of pathways.

Results: For the above-described analysis, small RNA sequencing data from public data repositories were used. Sequencing results revealed that hsa-miR-548ba was expressed at the highest level in the ovarian granulosa cells and uterine myometrial samples together with another twelve and one hsa-miR-548 family members, respectively. Pathway enrichment analysis of microRNA targets in the ovarian samples revealed the hsa-miR-548ba and hsa-miR548b-5p co-regulation of RAB geranylgeranylation in mural granulosa cells. Moreover, other hsa-mir-548 family members co-regulate pathways essential for ovarian functions (PIP3 activates AKT signalling and signalling by ERBB4). In addition to hsa-miR-548ba, hsa-miR-548o-3p is expressed in the myometrium, which separately targets the peroxisome proliferator-activated receptor alpha (PPARA) pathway.

Conclusion: This study reveals that hsa-mir-548 family members are expressed in variable combinations in the reproductive tract, where they potentially fulfil different regulatory roles. The results provide a reference for further studies of the hsa-mir-548 family role in the reproductive tract.
\end{abstract}

Keywords: Hsa-mir-548 family, Hsa-miR-548ba, Granulosa cells, Myometrium, FSHR

\section{Background}

MicroRNAs (miRNAs) are a class of non-coding RNA molecules $\sim 22$ nucleotides in length with an important role in post-transcriptional gene expression regulation [1]. miRNAs target genes via the Watson-Crick complementarity principle. The seed sequences of miRNAs, the 2-7 nucleotides positioned in the $5^{\prime}$ region, play an

\footnotetext{
* Correspondence: ilmatar.rooda@gmail.com

'Department of Chemistry and Biotechnology, Tallinn University of Technology, Akadeemia tee 15, 12618 Tallinn, Estonia

${ }^{2}$ Competence Centre on Health Technologies, Teaduspargi 13, 50411 Tartu, Estonia

Full list of author information is available at the end of the article
}

important role in the precise targeting of mRNA [2, 3], while other regions in the miRNA sequence complement the target's specificity [4]. Overall, miRNAs play wellestablished roles in gene expression regulation in normal and pathological conditions [5]. Moreover, different tissues demonstrate variable miRNA expression patterns that determine tissue characteristics, differentiation, and functions [6].

miRNAs are categorized into families according to the mature miRNA sequence and/or structure of their pre-miRNAs [7]. Mir-548 family miRNAs originate from the mariner-derived element 1 (Made1) transposable elements [8]: primate-specific short miniature

(c) The Author(s). 2021 Open Access This article is licensed under a Creative Commons Attribution 4.0 International License, which permits use, sharing, adaptation, distribution and reproduction in any medium or format, as long as you give appropriate credit to the original author(s) and the source, provide a link to the Creative Commons licence, and indicate if changes were made. The images or other third party material in this article are included in the article's Creative Commons licence, unless indicated otherwise in a credit line to the material. If material is not included in the article's Creative Commons licence and your intended use is not permitted by statutory regulation or exceeds the permitted use, you will need to obtain permission directly from the copyright holder. To view a copy of this licence, visit http://creativecommons.org/licenses/by/4.0/ The Creative Commons Public Domain Dedication waiver (http://creativecommons.org/publicdomain/zero/1.0/) applies to the data made available in this article, unless otherwise stated in a credit line to the data. 
inverted-repeat transposable elements (MITEs) that form almost perfect palindromes. The secondary structure of Made1 RNA contains highly stable hairpin loops that are recognized by the miRNAprocessing machinery [8]. Over the course of evolution, mir-548 family members have undergone several seed-shifting events, leading to changes in the seed sequences and hence the increased variability of their mRNA targets [9].

Human miRNA hsa-miR-548ba is a member of the mir-548 family and was originally described in granulosa cells of human pre-ovulatory follicles. The hsa-miR$548 \mathrm{ba}$ gene is located in the intronic region of the follicle-stimulating hormone receptor (FSHR) gene [10]. Hsa-miR-548ba target analysis has revealed PTEN and LIFR as its specific targets. Both of these genes play a well-established role in follicle activation and growth, indicating that hsa-miR-548ba may also have potential regulatory importance in follicle development [11].

Follicles are ovarian structures containing the oocyte and the supporting somatic cells: theca and granulosa cells, responsible for steroidogenesis and the metabolic support of the oocyte [12]. FSHR has important functions in follicle growth in the ovaries as well as sperm development in the testes [13]. By the time the follicle reaches the pre-ovulatory stage, granulosa cells have differentiated into cumulus and mural granulosa cell populations (CGC and MGC, respectively), and the follicle is filled with follicular fluid (FF) that physically separates these cell populations [12]. The main roles of CGC and MGC are providing essential metabolic support to the oocyte and steroid hormone production, respectively [12]. FSHR knock-out mice displayed disordered follicle growth and ovulation [14]. Similarly, point mutations in human FSHR result in arrested follicle development [15]. Therefore, disturbances in FSHR expression lead to female infertility [14, 15]. Analogously, Sertoli cells in the testes express FSHR, where FSH binding indirectly activates the proliferation of germ lineage cells. FSH also regulates the role of Sertoli cells as supporters of sperm cell development [16]. Male FSHR knock-out mice and humans with point mutations in the FSHR gene have decreased spermatogenesis rates and are subfertile [17].

In addition to the ovary and testis, FSHR expression is also detected in the following reproductive tissues: the endometrium [18] and myometrium [19] of the uterus, fallopian tube [20], and cervix [19]. The uterus is mainly composed of myometrial cells, the central roles of which are protecting the growing foetus and facilitating its delivery at the end of the pregnancy through muscular contractions [21, 22]. Myometrial smooth muscle cells express receptors for estrogen and progesterone important for myometrial cell growth and tissue activation during labour [21]. In addition, FSHR is present in the myometrium, where it may also participate in myometrial contractility [23]. The endometrium is a hormonally regulated inner lining of the uterus that is receptive for embryo implantation during only a short period of the menstrual cycle. During this period, the tissue develops specific functional and structural characteristics that allow the attachment of the embryo and its implantation [24, 25].

The aim of the current study is to understand the gene regulatory network between hsa-mir-548 family members that are co-expressed in a certain cell type or tissue. Distinguishing their unique and overlapping gene targets will allow a better interpretation of their importance in tissue function. Due to the importance of FSHR in folliculogenesis and the high level of expression of hsamiR-548ba in granulosa cells, we aimed to investigate the expression of all hsa-mir-548 members in the context of reproductive tissues where FSHR expression has been detected. We start by providing an update to the status of the hsa-mir-548 family according to the latest miRBase version [26]. Finally, we provide a model of potential co-regulation of mRNA targets and pathways between hsa-miR-548ba and other hsa-mir-548 family members in human reproductive tissues.

The mir-548 family is primate-specific. Members of this family are found in Homo sapiens, Pan troglodytes, Callithrix jacchus, Macaca mulatta, Pongo pygmaeus and Gorilla gorilla. MiR-548ba has been reported uniquely in Homo sapiens; therefore, this study focuses only on the members detected in humans and excludes all other primates.

\section{Results}

According to the full version history of miRBase, the first members of the hsa-mir-548 family were added into v9. The number of members has since been increasing with almost all new releases of miRBase in correlation with the detection of new miRNA sequences due to the increasing availability of RNA sequencing data. MiRBase v22.1 contains 86 mature human mir-548 family sequences (Fig. 1A). Hsa-miR-548ba is a relatively new member of the family, added into v20.

\section{Human mir-548 family distribution throughout the human genome}

The human mir-548 family contains several multi-copy pre-miRNAs in the genome, and as a result, different miRNA precursor sequences give rise to the same mature sequences of hsa-miR-548. For example, hsa-miR$548 \mathrm{f}$ and hsa-miR-548h have 5 different pre-miRNAs in the human genome. There are in total 76 different hsamir-548 pre-miRNA sequences, located throughout the human genome (Fig. 1B). The highest enrichment is observed on chromosomes 6,8 , and X. Two human 

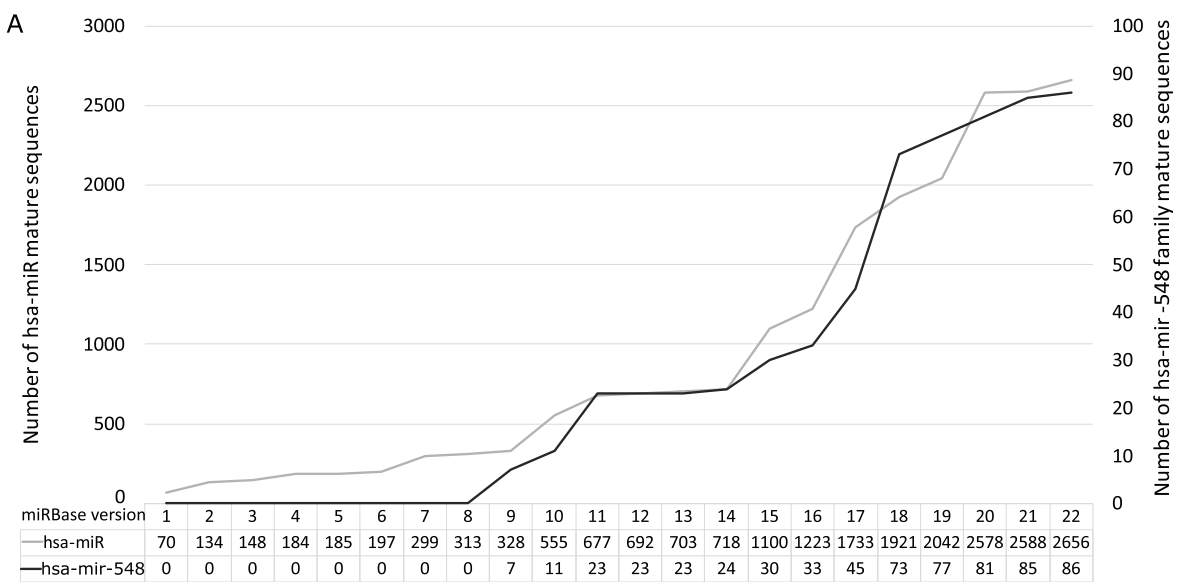

B

C

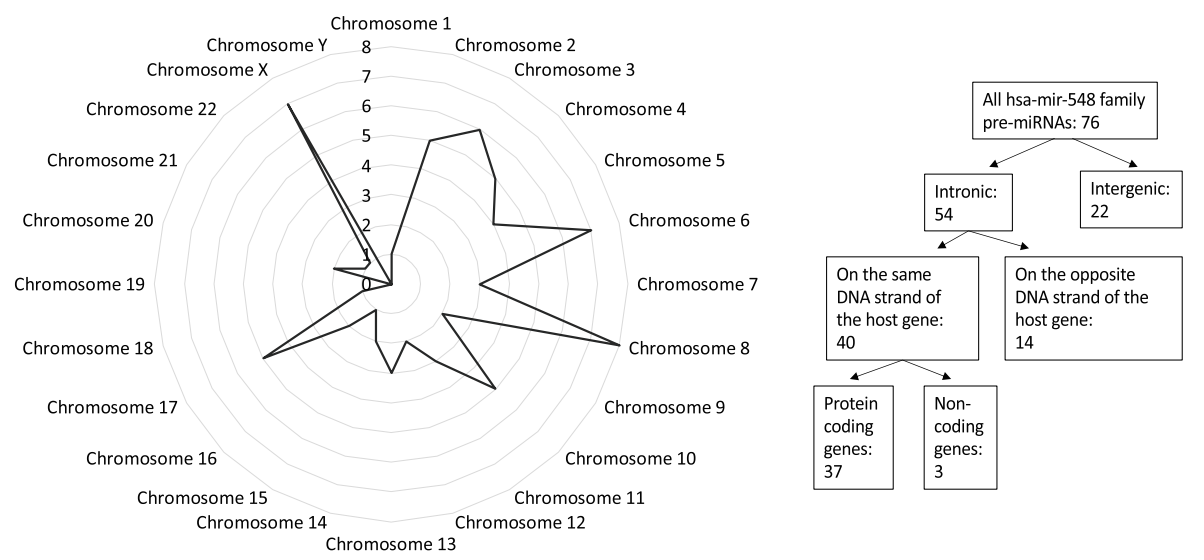

Fig. 1 Hsa-mir-548 family members in the miRBase database and in the human genome. (A) The number of all annotated human miRNA (grey line) and hsa-mir-548 family (black line) sequences in the full versions of the miRBase database. (B and C) Hsa-mir-548 family pre-miRNA sequences in the human genome: distribution by chromosome (B), distribution between the genomic loci (C)

chromosomes (19 and Y) lack hsa-mir-548 family sequences completely.

From the 76 hsa-mir-548 sequences, 54 are located in the intronic regions and 22 sequences are of intergenic origin (Fig. 1C). From 54 intronic miRNAs, 40 sequences were located on the same DNA strand as their host gene (Additional file 1 Supplementary Table S1). As 37 of these 40 are protein-coding genes, there is a potential co-transcription of the host gene and the corresponding intronic miRNA.

\section{Sequence similarity analysis of hsa-mir-548 family members}

Sequence similarity analyses were performed for both mature and pre-miRNA sequences. Shorter distances between mature sequences on the phylogenetic tree indicate higher conservation compared to pre-miRNA sequences (Additional file 2 Supplementary Fig. S1 and $\mathrm{S} 2$ ). The hsa-miR-548ba mature sequence displayed the shortest distances to the following miRNAs: hsa-miR-548 m, hsa-miR-548ag, hsa-miR-548d-5p, hsa-miR-548ay-5p, and hsa-miR-548ad-5p (Fig. 2C, Additional file 2 Supplementary Fig. S1). In addition, hsa-miR-548ag; hsa-miR548ai and hsa-miR-570-5p share the critical seed sequence with hsa-miR-548ba (Fig. 2D), although the two latter miRNAs demonstrate dissimilarities in their 3'part and therefore reside more distantly in the phylogenetic tree (Additional file 2 Supplementary Fig. S1).

Moreover, a sequence similarity analysis was performed between hsa-mir-548 family members and Made1, the MITE elements giving rise to these miRNAs (Additional file 2 Supplementary Fig. S3 and S4, respectively). HsamiR-548-5p sequences demonstrate higher conservation and similarity to Made1 compared to hsa-miR-548-3p sequences. This result confirms a previous similar observation [9]. Hsa-miR-548ba belongs to the hsa-miR-548-5p sequences and is therefore a more conserved family member. Hence, it is highly probable that hsa-miR-548ba and other hsa-mir-548 family members co-expressed in a tissue regulate a set of the same mRNA targets. 


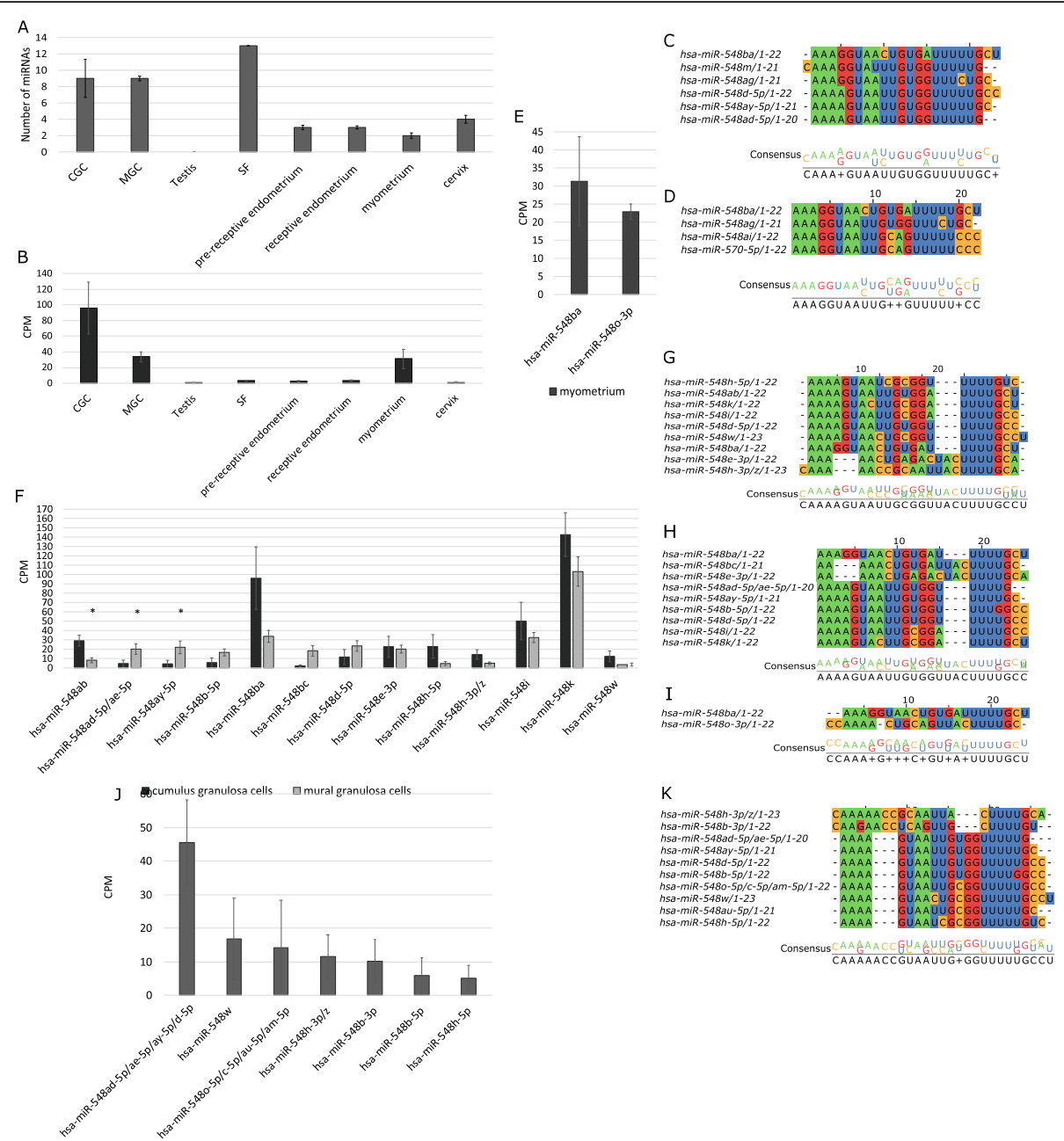

Fig. 2 The expression and sequence similarity of hsa-mir-548 family members in reproductive tissue samples. (A) The average number of hsa-mir-548 family members present in reproductive tissues with cut-off $>10$ counts per million (CPM). (B) The expression levels of hsa-miR-548ba in reproductive tissues. The sequencing results are displayed as a mean of CPM \pm SEM. CGC-cumulus granulosa cells $(n=3)$, MGC-mural granulosa cells ( $n=3)$, testis $(n=5)$, SF-seminal fluid $(n=1)$, pre-receptive endometrium $(n=12)$, receptive endometrium $(n=12)$, myometrium $(n=3)$, and cervix $(n=4)$. (C) The closest hsa-mir-548 family members to hsa-miR-548ba according to sequence similarity. (D) Hsa-mir-548 family members which share a seed sequence with hsa-miR-548ba. The sequence length in nucleotides is noted after the slash. (E) miRNAs from the hsa-miR-548 family expressed in the myometrium. (F) miRNAs of the hsa-miR-548 family expressed in cumulus and mural granulosa cells. The alignment of hsa-miR-548 family sequences co-expressed with hsa-miR-548ba in the analysed tissues: $\mathbf{G})$ cumulus granulosa cells; $(\mathbf{H})$ mural granulosa cells; (I) myometrium; (J) The expression of hsa-mir-548 family members in the cell-depleted follicular fluid of the ovarian follicle; (K) the alignment of extracellular miRNAs observed in follicular fluid. Expression levels are displayed as a mean of counts per million (CPM) \pm SEM). ${ }^{*} p<0.05$, Student's t-test

\section{Hsa-mir-548 family expression in reproductive tissues} In order to quantify the expression levels of the hsamiR-548 family members in human reproductive tissues, small RNA high-throughput sequencing results from male and female reproductive tissues were analysed (Table 1). Tissues were selected for analysis according to the availability of small RNA sequencing data and positivity for FSHR expression. From the male reproductive tissues, small RNA sequencing results were only available for the whole testis tissue homogenate and seminal fluid (SF). From the female reproductive tissues, data was available for $\mathrm{MGC}$, CGC, and FF of the ovary, myometrium, and endometrium from the uterus and cervix.

The highest number of hsa-mir-548 family members was detected from the SF, CGC, and MGC samples (cut-off $>10$ counts per million (CPM), Fig. 2A). From the testis samples, none of the hsa-mir-548 family members reached the set cut-off limit (Fig. 2A). The full lists of hsa-mir-548 family members expressed above >10 CPM cut-off level are presented in Additional file 1 Supplementary Table S2. 
Table 1 A description of data used in the hsa-mir-548 family analysis of reproductive tissues

\begin{tabular}{|c|c|c|c|c|c|c|}
\hline \multirow[t]{7}{*}{$\begin{array}{l}\text { Cellular } \\
\text { samples }\end{array}$} & $\begin{array}{l}\text { Tissue of } \\
\text { origin }\end{array}$ & Cell type & Data repository & $\begin{array}{l}\text { Accession } \\
\text { number }\end{array}$ & $\begin{array}{l}\text { Number of } \\
\text { samples }\end{array}$ & Description \\
\hline & Ovary & Granulosa & GEO & GSE46508 & 6 & $\begin{array}{l}\text { Two cell types of human granulosa cell } \\
\text { samples were obtained from } \\
\text { pre-ovulatory follicles: cumulus granulosa } \\
\text { (CGC) and mural granulosa (MGC) } \\
\text { collected from stimulated pre-ovulatory } \\
\text { follicles [10]. }\end{array}$ \\
\hline & Uterus & Endometrial tissue & GEO & GSE108966 & 24 & $\begin{array}{l}\text { Human endometrial samples were } \\
\text { collected from two time-points of the } \\
\text { same menstrual cycle: early-secretory } \\
\text { phase corresponding to pre-receptive } \\
\text { endometrium and mid-secretory phase } \\
\text { corresponding to receptive } \\
\text { endometrium [27]. }\end{array}$ \\
\hline & Uterus & Myometrium tissue & GEO & GSE100338 & 3 & [28] \\
\hline & Uterus & Cervix tissue & GEO & GSE145372 & 4 & [29] \\
\hline & Testis & Testis tissue & GEO, ENCODE & $\begin{array}{l}\text { ENCSR229WIW, } \\
\text { ENCSR626GVP } \\
\text { and GSE149084 }\end{array}$ & 5 & Whole testis tissue sections [30]. \\
\hline & $\begin{array}{l}\text { Reproductive } \\
\text { track }\end{array}$ & $\begin{array}{l}\text { Non-sperm cellular } \\
\text { fraction of seminal } \\
\text { fluid (SF) }\end{array}$ & GEO & GSE56686 & 1 & $\begin{array}{l}\text { Non-sperm cellular fraction of SF, which } \\
\text { includes prostatic epithelial, urothelial } \\
\text { and inflammatory cells [31]. }\end{array}$ \\
\hline \multirow[t]{2}{*}{$\begin{array}{l}\text { Extracellular } \\
\text { samples }\end{array}$} & $\begin{array}{l}\text { Tissue of } \\
\text { origin }\end{array}$ & Cell type & Data repository & $\begin{array}{l}\text { Accession } \\
\text { number }\end{array}$ & $\begin{array}{l}\text { Number of } \\
\text { samples }\end{array}$ & Description \\
\hline & Ovary & Follicular fluid (FF) & GEO & GSE157037 & 8 & $\begin{array}{l}\text { Extracellular miRNAs were extracted } \\
\text { from cell-depleted ovarian follicular } \\
\text { fluid (FF) from stimulated } \\
\text { pre-ovulatory follicles [32]. }\end{array}$ \\
\hline
\end{tabular}

The highest expression levels of hsa-miR-548ba were observed in ovarian CGC and MGC samples (Fig. 2B). Outside of the ovary, hsa-miR-548ba expression was the highest in myometrial tissue compared to the other samples. Testicular, SF, endometrial, and cervical samples demonstrated expression levels with borderline detection values (Fig. 2B).

\section{Hsa-mir-548 expression in granulosa cells and myometrium}

To further study the potential and significance of the post-transcriptional co-regulation effect that hsa-miR548ba may exhibit with its co-expressed family members, ovarian and myometrial samples were further analysed, as hsa-miR-548ba was only detected in these samples.

Sequencing results of granulosa cells revealed the expression of 13 different mature hsa-mir-548 family members (Fig. 2F). From those miRNAs, three (hsa-miR548ab, hsa-miR-548ad-5p/ae-5p and hsa-miR-548ay) were differentially expressed between MGC and CGC samples $(p<0.05)$.

The miRNAs which share the same seed sequence with hsa-miR-548ba (Fig. 2D) are not co-expressed in granulosa cells. However, sequence alignment results reveal that a number of miRNAs detected in granulosa cells share the seed sequence with each other (Fig. 2G-H). Specifically, hsa-miR-548ab, hsa-miR-548d-5p, hsa-miR-548 h-5p, hsamiR-548i, and hsa-miR-548w in CGC and hsa-miR-548ay, hsa-miR-548ae-5p, hsa-miR-548ad-5p, hsa-miR-548b-5p, hsa-miR-548d-5p and hsa-miR-548i in MGC contain the same seed sequences. Therefore, the co-regulation of common target genes by these miRNAs is possible and expected to occur in granulosa cells.

From miRNAs with the highest sequence similarity to hsa-miR-548ba, only hsa-miR-548ay-5p and hsa-miR548ad-5p are present in MGC, and hsa-miR-548d-5p in both CGC and MGC are expressed above the cut-off > 10 CPM (Fig. 2F-H).

Compared to granulosa cells, the myometrium expresses only two hsa-mir-548 family members above the > 10 CPM cut-off: hsa-miR-548ba and hsa-miR-548o-3p (Fig. 2E). HsamiR-548o-3p is evolutionarily distant from hsa-miR-548ba, and these two miRNAs do not share a common seed sequence (Fig. 2I). In addition to the myometrium, hsa-miR548o-3p is expressed in the endometrium, cervix, and SF samples (Additional file 1 Supplementary Table S2).

Extracellular hsa-mir-548 family miRNAs in the follicular fluid miRNAs are known to be present in the extracellular space as a part of RNA-binding protein (RBP) complexes 
or as loaded into extracellular vesicles (EV) [33]. However, the potential for the hsa-mir-548 family miRNAs to be secreted into extracellular spaces has not been studied. Due to the high expression levels of hsa-miR548ba and several other members of the family in the ovarian follicular somatic cells, the extracellular profile was determined from the example of the ovarian celldepleted FF [22], where seven family members were detected (50\% of samples > 10 CPM cut-off, Fig. 2J). We observed that the miRNAs expressed at the highest levels in the cellular samples (hsa-miR-548k, hsamiR-548ba and hsa-miR-548i) were not detected in FF. This suggests that those miRNAs are cell-specific and are not secreted into extracellular spaces. On the other hand, hsa-miR-548o-5p, hsa-miR-548c-5p, hsamiR-548am-5p, and hsa-miR-548b-3p in FF are not expressed in granulosa cells above the determined cut-off level (Fig. 2J).

Specific motifs in the 3' half of the miRNA sequence have the potential to determine whether miRNAs are secreted into the extracellular space or are retained in the cells: for example, GGAG and UGCA appear frequently in extracellular and cellular miRNAs, respectively [34]. In addition, the AGG motif may be involved in extracellular miRNA trafficking [35]. However, miRNAs present in FF samples do not contain GGAG nor AGG motifs (Fig. 2K). Cellular motif UGCA is present in hsa-miR$548 \mathrm{~h}-3 \mathrm{p} / \mathrm{z}$. The fact that those miRNAs are present in the extracellular space may be the result of non-specific secretion.

\section{The signature of hsa-mir-548 family expression is characteristic for each female reproductive tissue}

All ovarian follicle sample types form separate clusters according to their hsa-mir-548 family expression patterns (Fig. 3A-B). As expected, cellular and extracellular samples cluster separately. Moreover, two granulosa cell types form separate clusters according to their hsa-mir548 expression patterns (Fig. 3B). MGC and FF samples display more similar expression patterns compared to CGC cells (Fig. 3A-B). This may indicate that MGC is the primary source of hsa-mir-548 members secreted into FF as MGC is the most abundant somatic cell type inside the pre-ovulatory follicle.

In addition to granulosa cells, hsa-miR-548ba exhibited high expression levels in the myometrial tissue. For clustering analysis, all available uterine tissue samples (endometrium, myometrium and cervix) were compared. The results exhibited a characteristic hsa-mir-548 family expression pattern for endometrium, myometrium, and cervical samples (Fig. 3C-D). Endometrial samples of pre-receptive and receptive stages clustered together (Fig. 3C-D), indicating that hsa-mir-548 family expression levels do not significantly change upon acquiring endometrial receptivity. Moreover, myometrial and cervical samples form closer clusters compared to endometrial samples (Fig. 3C).

Overall, the clustering analysis illustrates that it is possible to distinguish female reproductive tissues and cell types by the expression signature of the hsa-mir-548 family members. Therefore, this miRNA family possesses regulatory roles specific to cell type.

\section{Pathways regulated by hsa-miR-548 members co- expressed in granulosa cells}

Since multiple hsa-mir-548 family members are coexpressed with hsa-miR-548ba in the ovarian granulosa cells, we investigated their tissue-specific potential for regulating common signalling pathways with relevance to female fertility. Target genes were predicted for all miRNAs expressed above >10 CPM cut-off level in CGC or MGC cells and the obtained lists were used as inputs for Reactome pathway enrichment analysis, the results of which are presented in Additional file 1 Supplementary Table S3. Target prediction results revealed that, despite the similarities between the sequences, miRNAs expressed in CGC or MGC target mostly individual genes with a small overlapping part (Fig. 4A and $\mathrm{B}$, respectively).

Pathway enrichment analysis of targeted genes concluded that hsa-miR-548ba does not co-regulate common pathways in CGC with other cell-type-specific hsa-miR-548 family members. In MGC, hsa-miR-548ba revealed the co-regulation of "RAB geranylgeranylation" pathway with hsa-miR-548b-5p. From the other hsamir-548 family members hsa-miR-548d-5p and hsa-miR548i co-regulate, "PIP3 activates the AKT signalling" pathway in both CGC and MGC. This pathway is additionally targeted by hsa-miR-548w and hsa-miR548b-5p in CGC and MGC, respectively. Additionally, "PI5P, PP2A, and IER3 regulate PI3K/AKT signalling" pathway is commonly regulated by hsa-miR-548d-5p and hsa-miR-548b-5p in MGC (Additional file 1 Supplementary Table S3A and S3B).

In the context of ovarian function, the above-mentioned pathways "PIP3 activates AKT signalling" and "PI5P, PP2A and IER3 regulate PI3K/AKT signalling" have been previously studied [36-38]. In addition, the "Translocation of SLC2A4 (GLUT4) to the plasma membrane" targeted by hsa-mir-548ba in both CGC and MGC, as well as "Signalling by ERBB4" targeted by hsa-miR-548b-5p in MGC, demonstrate the importance of the corresponding miRNAs in ovarian functions $[39,40]$.

\section{Pathways regulated by hsa-miR-548 members expressed in myometrium}

Although the myometrial cells exhibited the expression of only two hsa-mir-548 family members (hsa-miR- 

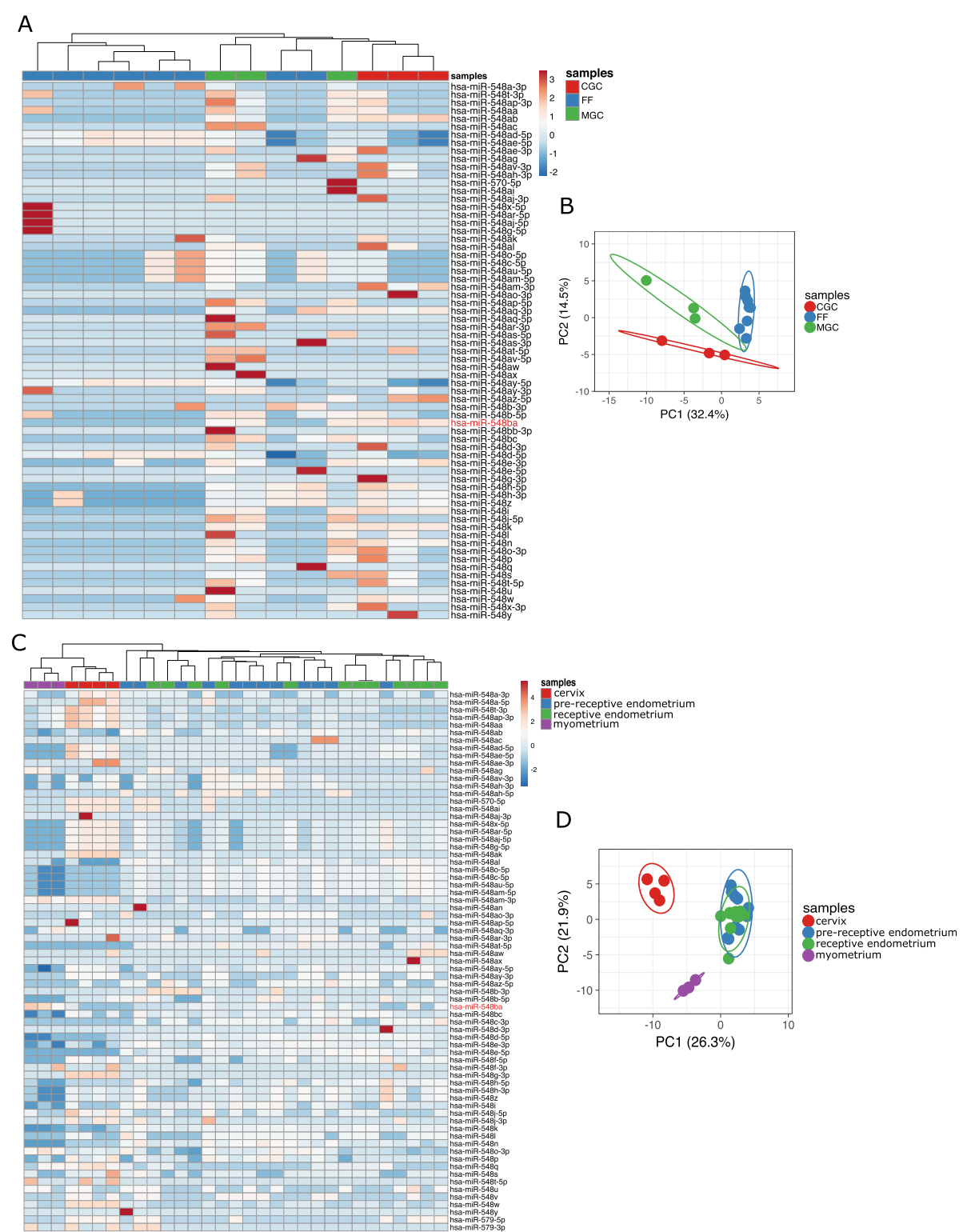

Fig. 3 Expression levels of hsa-mir-548 family miRNAs in the human ovarian follicle and uterine samples. (A) A heatmap of hsa-mir-548 family expression levels in individual ovarian samples; (B) a PCA plot of ovarian follicle cellular and extracellular samples according to the expression levels of hsa-mir-548 family miRNAs; (C) a heatmap of hsa-mir-548 family expression levels in individual uterine samples; (D) a PCA plot of uterine samples according to the expression levels of hsa-mir-548 family levels. FF-cell-depleted follicular fluid, CGC-cumulus granulosa cells, MGC-mural granulosa cells. The location of hsa-miR-548ba on the heatmap is highlighted in red. The heatmap colour scale displays $\ln (x+1)$ transformed CPM values

548ba and hsa-miR-548o-3p), a commonly regulated pathway "Signalling by BRAF and RAF fusions" by these two miRNAs was detected.

In the context of myometrial functions, the following important regulatory pathways were targeted by hsamiR-548o-3p: "PI5P, PP2A and IER3 regulate PI3K/AKT signalling" and "PPARA activates gene expression".

\section{Discussion}

Hsa-mir-548 is a primate-specific miRNA family derived from Made1 transposable element [8]. Made1 elements are MITEs with genomic locations either close to or within genes, where they may be involved in gene regulation [41]. Hsa-mir-548 family members are transcribed from most human chromosomes, while some other miRNA families exhibit chromosome-specific locations in the genome [7]. The distribution analysis of hsa-mir548 family members in the human genome exhibited that the majority of pre-miRNA sequences (54/76) are located in the intronic regions of genes. This is in an accordance with the preferable genome locations of MITEs [41]. Moreover, chromosome Y, which contains the 

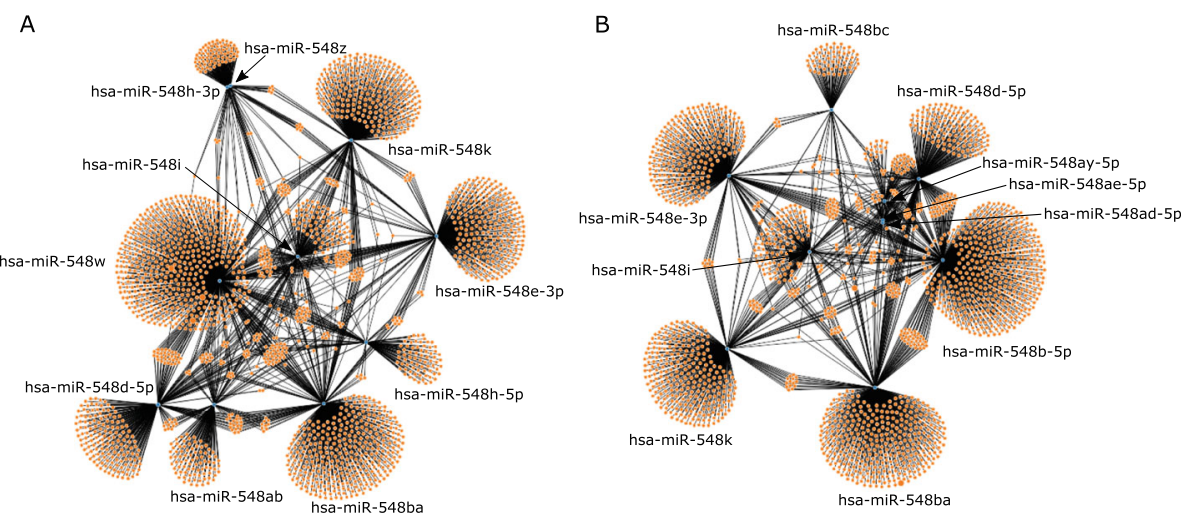

Fig. 4 Target prediction for hsa-mir-548 family miRNAs expressed in cumulus granulosa cells (A) and mural granulosa cells (B). Each orange node represents one target gene, and genes targeted by more than one miRNA are connected with an edge

smallest number of genes compared to other chromosomes [42], does not contain any hsa-mir-548 members. However, gene-rich chromosome 19 [43] likewise did not contain any hsa-mir-548 miRNA sequences. It is possible that not all hsa-mir-548 family members have been discovered. Since the first sequence of hsa-mir-548 was included to miRBase, new sequences have been added to almost every new miRBase release, correlating with the rapid development and reduced cost of highthroughput sequencing technologies. Moreover, using a bioinformatic approach, 34 additional precursor sequences of hsa-mir-548 have been discovered, indicating that this family could be larger [44]. However, their expression still needs experimental validation.

miRNAs are important gene expression regulators in both the male and female reproductive tissues and aberrant miRNA expression can lead to infertility $[45,46]$. Hsa-miR-548ba expression analysis in reproductive samples revealed that this particular miRNA is expressed at the highest level in granulosa cells, where it was first discovered. It has been previously shown that the expression pattern of hsa-miR-548ba is similar to that of FSHR and FSH treatment upregulates hsa-miR548ba expression levels in human granulosa cells [11]. Human ovarian granulosa cells [13], endometrium [47], myometrium [19], cervix [19] and testis Sertoli cells [13] all express FSHR. In addition to granulosa cells, hsa-miR-548ba exhibited high expression levels in the myometrial tissue. These results reveal the tissue-specific expression of hsa-miR-548ba that may be derived from a different miRNA expression regulation than that observed in the granulosa cells of its host gene, FSHR. However, differences in expression level may also be caused by technical errors. The datasets used for this study were obtained from data repositories and, therefore, RNA extraction and library preparation were not universal for all samples. This may be the cause of the lower expression of hsa-miR-548ba in the endometrium, cervix, and testis samples.

Overall, granulosa cells express 13 members of the hsa-mir-548 family. From those, miRNAs hsa-miR548ab, hsa-miR-548ad-5p/ae-5p, and hsa-miR-548ay-5p were differentially expressed between MGC and CGC samples ( $p$-value <0.05). Although detected in a few other human sample types (hsa-miR-548ab expression is reported in human B-cells [48], hsa-miR-548ad-5p/ae-5p and hsa-miR-548ay-5p are present in synovial tissue samples [49], and hsa-miR-548ad-5p is present in blood plasma samples), the roles of these specific miRNAs have not been investigated. However, in our samples, hsa-miR-548ad-5p was detected in extracellular FF as one of the most abundant miRNAs. According to the comparisons in ovarian datasets, it can be deduced that hsa-miR-548ad-5p is secreted from MGC and may be involved in intercellular signalling in the follicle. Therefore, the investigated miRNA family potentially has unknown importance in follicular function.

miRNAs which share the same seed sequence with hsa-miR-548ba are not co-expressed in granulosa cells, which indicates that this miRNA potentially has an individual specific regulatory role in the ovary. However, despite this, some targets were shared between other coexpressed members of the hsa-mir-548 family with different seed sequences. It has been well established that one mRNA can be targeted by multiple miRNAs [50]. Moreover, target prediction algorithms like miRWalk use additional features to miRNA seed sequence for target prediction [51]. The hsa-mir-548 family has gone through several seed-shifting events, which has resulted in various seed sequences in the members [9]. Different seed sequence variants were also present in miRNAs expressed in ovarian and myometrial samples. Nevertheless, some hsa-mir-548 family miRNAs expressed in granulosa cells have common seed sequences and 
consequently overlap with part of the predicted target genes; for example, hsa-miR-548b-5p, hsa-miR-548d-5p, and hsa-miR-548i expressed in MGCs.

The majority of enriched pathways were targeted by different individual miRNAs in the granulosa cells. All together there were three exceptions. The first exception was the "PIP3 activates AKT signaling" pathway, which is targeted by hsa-miR-548d-5p and hsa-miR-548i in CGC and MGC and additionally targeted by hsa-miR548w and hsa-miR-548b-5p in CGC and MGC, respectively. This pathway is involved in regulating the balance between dormancy and activation of follicles, granulosa cell differentiation, and proliferation [38]. The second exception was the "PI5P, PP2A, and IER3 regulate PI3K/ AKT signalling" pathway targeted by hsa-miR-548d-5p in CGC and MGC, and hsa-miR-548b-5p in MGC. IER3 is a part of a gonadotropin-EGR2-IER3 axis with a role in granulosa cell survival during follicle development [36]. Additionally, PP2A participates in the regulation of PKC-mediated inflammation in rat granulosa cells [37]. The last co-regulated pathway is " $\mathrm{RAB}$ geranylgeranylation" targeted by hsa-miR-548ba and hsa-miR-548b-5p in MGC. The depletion of the geranylgeranylation substrate geranylgeranyl diphosphate (GGPP) in mice oocytes inhibits Rab27a geranylgeranylation, which is required for Rab protein activation. Rab27a plays a possible role in oocyte protein secretion. Therefore, disturbances in this pathway impair oocyte-granulosa cell communication, which is necessary for normal follicle development [52].

Pathways targeted by individual hsa-mir-548 members have additional known roles in granulosa cells. For example, hsa-miR-548ba targets the "translocation of SLC2A4 (GLUT4) to the plasma membrane" pathway. GLUT4 is involved in glycose uptake and FSH stimulates this process in granulosa cells [39]. Granulosa cells of polycystic ovarian syndrome patients have a tendency to display abnormal glycose metabolism. Therefore, normal glycose metabolism is important for granulosa cell function [39]. Hsa-miR-548b-5p targets "Signalling by ERBB4" in MGC. ERBB4 plays a role in normal follicle development and disturbances in ERBB4 levels may lead to ovarian dysfunction [40]. To conclude, in addition to hsa-miR-548ba, other hsa-mir-548 family members regulate pathways important for granulosa cell functions.

Pathways "PIP3 activates AKT signalling" and "PI5P, PP2A and IER3 Regulate PI3K/AKT signalling" are targeted by miRNAs which share the seed sequences (hsa-miR-548d-5p, hsa-miR-548b-5p, hsa-miR-548i, and hsa-miR-548w). However, additional family members expressed in granulosa cells have the same seed sequence but do not target those pathways. Alignment results of miRNAs present in granulosa cells and alignment of the whole miRNA family demonstrated that, in addition to seed shifting events, nucleotide substitutions are present in miRNA sequences. These molecular events have changed potential targeting features [4] and have led to different target genes between miRNAs with the same seed sequence.

Myometrial samples express two hsa-mir-548 members: hsa-miR-548ba and hsa-miR-548o-3p. Both miRNAs regulate one common pathway: "Signalling by BRAF and RAF fusions". BRAF and RAF fusion is a result of chromosomal rearrangement events and is detected in distinct cancer types [53]. Therefore, in normal myometrial tissue, this pathway is not present. In addition, hsa-miR-548o-3p targets the "PI5P, PP2A, and IER3 regulate PI3K/AKT signalling" and "PPARA activates gene expression" pathways. From the first targeted pathway, PP2A regulates proteins involved in smooth muscle contraction [54]. In the second pathway, PPARA levels increase in the late pregnancy myometrium (gestation range 20-35 weeks) compared to nonpregnant women and decrease by the time of labour, suggesting that PPARA plays a role in maintaining pregnancy [55]. This indicates that the hsa-mir-548 family may have a regulatory role in myometrial gene expression regulation involved in contractile functions. Moreover, hsa-miR548o-3p expression was not detected in ovarian samples but was present in endometrial and cervical samples, confirming its organ-specific expression.

miRNAs have been detected from all body fluids, including FF [32, 56], and may be involved in cell-to-cell communication [33]. miRNAs can be secreted from cells as a part of RBPs or packed into EVs [33]. Many possible sorting mechanisms are proposed for loading miRNAs into EVs: sequence characteristics, post-transcriptional modifications, subcellular location, and intracellular concentration [33]. In this study, FF which contains both RBPs and EVs, was searched for hsa-mir-548 family members. As a result, 7 miRNAs were detected in FF. Some of the miRNAs were only detected in FF and not in granulosa cells, for example, hsa-miR-548o-5p and hsa-miR-548c-5p. miRNAs expressed at the highest levels in cellular samples were not present in FF, indicating that the secretion mechanism is not based on the intercellular concentration of miRNA molecules. miRNAs present in extracellular samples were aligned and a possible export motif was searched for. Known miRNA secretion motifs GGAG [34] and AGG [35] are not present in the miRNA sequences of hsa-mir-548 family members present in FF. Nevertheless, hsa-mir-548 family members have been detected from other body fluids in addition to FF: hsa-miR-548b-5p, hsa-miR-548c-5p and hsa-miR-548i [57], and hsa-miR-548a-3p [58] in blood serum samples, hsa-miR-548b-3p in blood plasma, bronchial lavage and peritoneal fluid, and hsa-miR-548d$5 \mathrm{p}$ in amniotic fluid [59]. Some miRNAs infiltrate into 
the FF from blood plasma [32], explaining the lack of their expression in the granulosa cells. Additionally, the oocyte has not been investigated as the source of miRNAs secreted into the FF due to the lack of such human data. Therefore, hsa-mir-548 family members are secreted into extracellular space by other cell types as well as ovarian granulosa cells. The mechanism by which hsa-mir-548 family members are selected for secretion remains unknown.

\section{Conclusion}

From all the analysed FSHR-positive samples, hsa-miR548ba transcribed from the intronic region of FSHR gene can be detected in the ovarian granulosa cells and the myometrium. This suggests that the expression of hsa-miR-548ba and FSHR are differently co-regulated in other FSHR-positive tissues. In addition to hsa-miR548ba, twelve and one other hsa-mir-548 family members are expressed in granulosa and myometrium samples, respectively. Moreover, hsa-mir-548 family members are detectable from the extracellular ovarian FF. miRNA target pathway enrichment analysis revealed that hsa-miR-548ba and hsa-miR-548b-5p co-regulate the RAB geranylgeranylation pathway in MGC. Disturbances in this pathway impair oocyte-granulosa cell communication. In addition to hsa-miR-548ba, other family members separately regulate essential pathways for granulosa cell function (PIP3 activates AKT signalling and signalling by ERBB4). This reveals that hsa-mir548's family regulatory role in granulosa cells is wider than previously acknowledged. Moreover, hsa-miR548o-3p expressed in myometrium targets the PPARA pathway which is associated with the maintenance of pregnancy. Furthermore, hsa-miR-548o-3p presents uterine-specific expression as it was detected only in myometrial, endometrial and cervical samples. Overall, hsa-mir-548 family members may play regulatory roles in ovarian follicle activation, development, granulosa cell differentiation, and proliferation. In the myometrium, the hsa-mir-548 family was predicted to regulate myometrial contractility and has a potential importance in the maintenance of pregnancy.

\section{Methods}

\section{Hsa-mir-548 family members and sequences}

The analysis of hsa-mir-548 family member curation was performed using the miRBase database [26]. Information about miRNA mature sequences was downloaded from all full miRBase versions with the exception of v22.1, which is the current release. Genomic locations of pre-miRNA sequences in the human genome were obtained from NCBI Gene [60] and Ensembl [61] databases.

Mature and pre-miRNA sequences were aligned in Jalview (v2.11.0) [62] using the Clustal Omega algorithm with standard settings [63]. Phylogenetic trees of mature and pre-miRNA sequences were constructed with the neighbour-joining method [64] from reads aligned with Clustal Omega in Jalview.

\section{Hsa-mir-548 family expression in human reproductive tissues}

All sequencing data used in the analyses were previously published and available in open data repositories (Table 1). From all available data, only samples from healthy control subjects were used. All miRNA raw FASTQ files were quality-filtered with Trimmomatic v0.39 [65] with the options of SLIDINGWINDOW:2: 20. Adapter sequences were removed and reads below 17 nucleotides in length were discarded. The remaining filtered and trimmed reads were counted and mapped to the primary assembly of human genome GRCh38 and annotated miRNA sequences from miRBase v22.1 using miRDeep2 with standard settings [66]. All miRNA raw counts obtained from miRDeep2 results were normalized to counts per million (CPM) using the edgeR package v.3.28.1 [67]. miRNA results were filtered by expression levels, and the cut-off was set $>10$ CPM for all cellular samples. The cut-off for extracellular samples was set to >10 CPM in 50\% of samples. Data visualization on heatmap and PCA plots was performed in ClustVis [68]. Statistical significance between CGC and MGC was calculated via a twotailed Student's t-test. The statistical significance level was set at $p<0.05$.

\section{Target prediction and gene ontology analysis}

Target genes were predicted for miRNAs with an expression cut-off level of $>10$ CPM with miRWalk version 3 [51]. Obtained miRNA target lists were input for gene enrichment analysis with miRWalk pathway analysis tool, and a statistical significance threshold was set at Benjamini-Hochberg FDR $<0.1$.

\section{Abbreviations}

CGC: cumulus granulosa cells; CPM: counts per million; EV: extracellular vesicles; FF: follicular fluid; FSHR: follicle-stimulating hormone receptor; Made: marinerderived element 1; MGC: mural granulosa cells; MITEs: miniature inverted-repeat transposable elements; RBP: RNA-binding protein; SF: seminal fluid

\section{Supplementary Information}

The online version contains supplementary material available at https://doi. org/10.1186/s12863-021-00997-w.

Additional file 1: Supplementary Table S1. Human hsa-mir-548 family pre-miRNA sequence locations in human genome. Supplementary Table S2. Hsa-mir-548 family miRNAs expressed in reproductive tissues. Supplementary Table S3. Reactome pathways of predicted miRNA targets

Additional file 2: Supplementary Fig. S1. Phylogenetic tree of mature sequences of hsa-mir-548 family members. Supplementary Fig. S2.

Phylogenetic tree of pre-miRNA sequences of miR-548 family members. 
Supplementary Fig. S3. Phylogenetic tree of Made1 and hsa-mir-548 family members. Supplementary Fig. S4. The alignment of Made1 and hsa-mir-548 family mature sequences

\section{Acknowledgments}

Not applicable.

\section{Authors' contributions}

$I R, M L, O P S, A S$, and AVM contributed to the study design; IR, BK and AVM analyzed the data. All authors were involved in compiling the manuscript and approved the final version.

\section{Funding}

This work was financially supported by grants from the Enterprise Estonia (grant EU48695); Estonian Research Council grants PSG433, PRG1076 and PSG608; Horizon 2020 innovation (ERIN) (grant no. EU952516) of the European Commission and by the Tallinn University of Technology development program 2016-2022, project code 2014-2020.4.01.16-0032. The funding bodies played no role in the design of the study and collection, analysis, and interpretation of data and in writing the manuscript.

\section{Availability of data and materials}

The datasets analysed during the current study are available in the Gene Expression Omnibus under accession numbers: GSE46508 (https://www.ncbi. nlm.nih.gov/geo/query/acc.cgi?acc=GSE46508), GSE108966 (https://www. ncbi.nlm.nih.gov/geo/query/acc.cgi?acc=GSE108966), GSE100338 (https:// www.ncbi.nlm.nih.gov/geo/query/acc.cgi?acc=GSE100338), GSE145372 (https://www.ncbi.nlm.nih.gov/geo/query/acc.cgi?acc=GSE145372), GSE149084 (https://www.ncbi.nlm.nih.gov/geo/query/acc.cgi?acc=GSE149084 ), GSE56686 (https://www.ncbi.nlm.nih.gov/geo/query/acc.cgi?acc=GSE56686) and GSE157037 (https://www.ncbi.nlm.nih.gov/geo/query/acc.cgi?acc=GSE15 7037) and ENCODE repository under accession numbers: ENCSR229WIW (https://www.encodeproject.org/experiments/ENCSR229WIW/) and ENCS R626GVP (https://www.encodeproject.org/experiments/ENCSR626GVP/).

\section{Declarations}

Ethics approval and consent to participate

Not applicable.

\section{Consent for publication}

Not applicable.

\section{Competing interests}

The authors declare that the research was conducted in the absence of any commercial or financial relationships that could be construed as a potentia conflict of interest.

\section{Author details}

'Department of Chemistry and Biotechnology, Tallinn University of Technology, Akadeemia tee 15, 12618 Tallinn, Estonia. ${ }^{2}$ Competence Centre on Health Technologies, Teaduspargi 13, 50411 Tartu, Estonia. ${ }^{3}$ Division of Obstetrics and Gynecology, Department of Clinical Science, Intervention and Technology (CLINTEC), Karolinska Institutet, 14186 Stockholm, Sweden. ${ }^{4}$ Department of Obstetrics and Gynecology, Institute of Clinical Medicine, University of Tartu, L. Puusepa St. 8, 50406 Tartu, Estonia. ${ }^{5}$ Institute of Genomics, University of Tartu, Riia 23b, 51010 Tartu, Estonia.

\section{Received: 3 May 2021 Accepted: 27 September 2021}

Published online: 08 October 2021

\section{References}

1. Bartel DP. MicroRNAs: genomics, biogenesis, mechanism, and function. Cell. 2004 Jan 23;116(2):281-97. https://doi.org/10.1016/S0092-8674(04)00045-5.

2. Lewis BP. Shih I-hung, Jones-Rhoades MW, Bartel DP, Burge CB. Prediction of Mammalian MicroRNA Targets Cell. 2003 Dec 26;115(7):787-98. https:// doi.org/10.1016/s0092-8674(03)01018-3.

3. Bartel DP. MicroRNA target recognition and regulatory functions. Cell. 2009 Jan 23;136(2):215-33. https://doi.org/10.1016/j.cell.2009.01.002.
4. Ding J, Li X, Hu H. TarPmiR: a new approach for microRNA target site prediction. Bioinformatics. 2016 Sep 15;32(18):2768-75. https://doi.org/10.1 093/bioinformatics/btw318.

5. Sereno M, Videira M, Wilhelm I, Krizbai IA, Brito MA. miRNAs in Health and Disease: A Focus on the Breast Cancer Metastatic Cascade towards the Brain. Cells [Internet]. 2020 Jul 28 [cited 2020 Dec 15];9(8). Available from: https://www.ncbi.nlm.nih.gov/pmc/articles/PMC7463742/

6. Ludwig N, Leidinger P, Becker K, Backes C, Fehlmann T, Pallasch C, et al. Distribution of miRNA expression across human tissues. Nucleic Acids Res. 2016 May 5;44(8):3865-77. https://doi.org/10.1093/nar/gkw116.

7. Kamanu TKK, Radovanovic A, Archer JAC, Bajic VB. Exploration of miRNA families for hypotheses generation. Sci Rep [Internet]. 2013 Oct 15 [cited 2020 Dec 15];3. Available from: https:/www.ncbi.nlm.nih.gov/pmc/articles/ PMC3796740/

8. Piriyapongsa J, Jordan IK. A Family of Human MicroRNA Genes from Miniature Inverted-Repeat Transposable Elements. PLoS One [Internet]. 2007 Feb 14 [cited 2020 Dec 15];2(2). Available from: https://www.ncbi.nlm.nih. gov/pmc/articles/PMC1784062/

9. Liang T, Guo L, Liu C. Genome-Wide Analysis of mir-548 Gene Family Reveals Evolutionary and Functional Implications I Biomed Biotechnol [Internet]. 2012 [cited 2020 Dec 15];2012 Available from: https://www.ncbi. nlm.nih.gov/pmc/articles/PMC3468316/

10. Velthut-Meikas A, Simm J, Tuuri T, Tapanainen JS, Metsis M, Salumets A. Research resource: small RNA-seq of human granulosa cells reveals miRNAs in FSHR and aromatase genes. Mol Endocrinol. $2013 \mathrm{Jul} ; 27(7): 1128-41$. https://doi.org/10.1210/me.2013-1058.

11. Rooda I, Hensen K, Kaselt B, Kasvandik S, Pook M, Kurg A, et al. Target prediction and validation of microRNAs expressed from FSHR and aromatase genes in human ovarian granulosa cells. Sci Rep. 2020 Feb 10; 10(1):2300. https://doi.org/10.1038/s41598-020-59186-x.

12. Hennet ML, Combelles CMH. The antral follicle: a microenvironment for oocyte differentiation. Int J Dev Biol. 2012;56(10-12):819-31. https://doi. org/10.1387/ijdb.120133cc.

13. Simoni M, Gromoll J, Nieschlag E. The follicle-stimulating hormone receptor: biochemistry, molecular biology, physiology, and pathophysiology*. Endocr Rev. 1997 Dec 1:18(6):739-73. https://doi.org/10.1210/edrv.18.6.0320.

14. Danilovich N, Babu PS, Xing W, Gerdes M, Krishnamurthy H, Sairam MR. Estrogen deficiency, obesity, and skeletal abnormalities in follicle-stimulating hormone receptor knockout (FORKO) female mice**this investigation was supported in part by the Canadian Institutes of Health Research. Endocrinology. 2000 Nov 1;141(11):4295-308. https://doi.org/10.1210/ endo.141.11.7765.

15. Aittomäki K, Lucena JL, Pakarinen P, Sistonen P, Tapanainen J, Gromoll J et al. Mutation in the follicle-stimulating hormone receptor gene causes hereditary hypergonadotropic ovarian failure. Cell. 1995 Sep 22;82(6):959-68. https://doi.org/10.1016/0092-8674(95)90275-9.

16. Petersen C, Söder O. The Sertoli cell - a hormonal target and 'super' nurse for germ cells that determines testicular size. HRP. 2006;66(4):153-61. https://doi.org/10.1159/000094142.

17. Oduwole OO, Peltoketo H, Huhtaniemi IT. Role of Follicle-Stimulating Hormone in Spermatogenesis. Front Endocrinol (Lausanne) [Internet]. 2018 Dec 14 [cited 2021 Jan 7];9. Available from: https:/www.ncbi.nlm.nih.gov/ pmc/articles/PMC6302021/

18. Ponikwicka-Tyszko D, Chrusciel M, Stelmaszewska J, Bernaczyk P, Sztachelska M, Sidorkiewicz I, et al. Functional Expression of FSH receptor in Endometriotic lesions. The Journal of Clinical Endocrinology \& Metabolism. 2016 Jul 1;101(7):2905-14. https://doi.org/10.1210/jc.2016-1014.

19. Stilley JAW, Christensen DE, Dahlem KB, Guan R, Santillan DA, England SK, et al. FSH receptor (FSHR) expression in human extragonadal reproductive tissues and the developing placenta, and the impact of its deletion on pregnancy in mice. Biol Reprod. 2014 Sep;91(3):74. https://doi.org/10.1095/ biolreprod.114.118562.

20. Zheng W, Magid MS, Kramer EE, Chen YT. Follicle-stimulating hormone receptor is expressed in human ovarian surface epithelium and fallopian tube. Am J Pathol. 1996 Jan;148(1):47-53.

21. Ono M, Bulun SE, Maruyama T. Tissue-Specific Stem Cells in the Myometrium and Tumor-Initiating Cells in Leiomyoma. Biol Reprod [Internet]. 2014 Dec [cited 2021 Jan 25];91(6). Available from: https://www. ncbi.nlm.nih.gov/pmc/articles/PMC4434985/

22. Shynlova O, Tsui $P$, Jaffer $S$, Lye SJ. Integration of endocrine and mechanical signals in the regulation of myometrial functions during 
pregnancy and labour. European Journal of Obstetrics \& Gynecology and Reproductive Biology. 2009 May 1;144:S2-10. https://doi.org/10.101 6/j.ejogrb.2009.02.044

23. Chrusciel M, Ponikwicka-Tyszko D, Wolczynski S, Huhtaniemi I, Rahman NA Extragonadal FSHR Expression and Function-Is It Real? Front Endocrinol [Internet]. 2019 [cited 2021 Jan 28];10. Available from: https://www. frontiersin.org/articles/10.3389/fendo.2019.00032/full\#B16

24. Riesewijk A, Martín J, van Os R, Horcajadas JA, Polman J, Pellicer A, et al. Gene expression profiling of human endometrial receptivity on days $\mathrm{LH}+2$ versus LH+7 by microarray technology. Mol Hum Reprod. 2003 May 1;9(5): 253-64. https://doi.org/10.1093/molehr/gag037.

25. Wang W, Vilella F, Alama P, Moreno I, Mignardi M, Isakova A, et al. Single-cell transcriptomic atlas of the human endometrium during the menstrual cycle. Nat Med. 2020 Oct;26(10):1644-53. https://doi.org/10.1038/s41591-020-1040-z.

26. Kozomara A, Birgaoanu M, Griffiths-Jones S. miRBase: from microRNA sequences to function. Nucleic Acids Res. 2019 Jan 8;47(D1):D155-62. https://doi.org/10.1093/nar/gky1141.

27. Rekker K, Altmäe S, Suhorutshenko M, Peters M, Martinez-Blanch JF, Codoñer FM, et al. A Two-Cohort RNA-seq Study Reveals Changes in Endometrial and Blood miRNome in Fertile and Infertile Women. Genes (Basel) [Internet]. 2018 Nov 23 [cited 2020 Dec 20];9(12). Available from: https://www.ncbi.nlm.nih.gov/pmc/articles/PMC6315937/

28. Chuang T-D, Khorram O. Expression profiling of IncRNAs, miRNAs, and mRNAs and their differential Expression in leiomyoma using nextgeneration RNA sequencing. Reprod Sci. 2018 Feb;25(2):246-55. https://doi. org/10.1177/1933719117711265.

29. Xu J, Zou J, Wu L, Lu W. Transcriptome analysis uncovers the diagnostic value of miR-192-5p/HNF1A-AS1NIL1 panel in cervical adenocarcinoma. Sci Rep [Internet]. 2020 Oct 6 [cited 2021 Jan 13];10. Available from: https:// www.ncbi.nlm.nih.gov/pmc/articles/PMC7538942/

30. An Integrated Encyclopedia of DNA Elements in the Human Genome. Nature. 2012 Sep 6:489(7414):57-74.

31. Selth LA, Roberts MJ, Chow CWK, Marshall VR, Doi SAR, Vincent AD, et al. Human seminal fluid as a source of prostate cancer-specific microRNA biomarkers. Endocr Relat Cancer. 2014 Aug 1;21(4):L17-21. https://doi.org/1 0.1530/ERC-14-0234.

32. Rooda I, Hasan MM, Roos K, Viil J, Andronowska A, Smolander O-P, et al. Cellular, Extracellular and Extracellular Vesicular miRNA Profiles of PreOvulatory Follicles Indicate Signaling Disturbances in Polycystic Ovaries. Int J Mol Sci. 2020 Dec;15, 21(24).

33. Fritz JV, Heintz-Buschart A, Ghosal A, Wampach L, Etheridge A, Galas D, et al. Sources and functions of extracellular small RNAs in human circulation. Annu Rev Nutr. 2016 Jul 17;36(1):301-36. https://doi.org/10.1146/annurevnutr-071715-050711.

34. Villarroya-Beltri C, Gutiérrez-Vázquez C, Sánchez-Cabo F, Pérez-Hernández D, Vázquez J, Martin-Cofreces N, et al. Sumoylated hnRNPA2B1 controls the sorting of miRNAs into exosomes through binding to specific motifs. Nat Commun. 2013 Dec 20;4(1):2980. https://doi.org/10.1038/ncomms3980.

35. Lee H, Li C, Zhang Y, Zhang D, Otterbein LE, Jin Y. Caveolin-1 selectively regulates microRNA sorting into microvesicles after noxious stimuli. J Exp Med. 2019 Sep 2;216(9):2202-20. https://doi.org/10.1084/jem.20182313.

36. Jin H, Won M, Shin E, Kim H-M, Lee K, Bae J. EGR2 is a gonadotropininduced survival factor that controls the expression of IER3 in ovarian granulosa cells. Biochem Biophys Res Commun. 2017 Jan 22;482(4):877-82. https://doi.org/10.1016/j.bbrc.2016.11.127.

37. Lin Y-Y, Sun D, Wu Y-L. Novel regulation of PKC-induced inflammation by Akt and protein phosphatase 2A in ovarian granulosa cells. Chin J Physiol. 2020 Aug;63(4):179-86. https://doi.org/10.4103/CJP.CJP_44_20.

38. Makker A, Goel MM, Mahdi AA. PI3K/PTEN/Akt and TSC/mTOR signaling pathways, ovarian dysfunction, and infertility: an update. J Mol Endocrinol. 2014 Dec 1;53(3):R103-18. https://doi.org/10.1530/JME-14-0220.

39. Anjali G, Kaur S, Lakra R, Taneja J, Kalsey GS, Nagendra A, et al. FSH stimulates IRS-2 expression in human granulosa cells through CAMP/SP1, an inoperative FSH action in PCOS patients. Cell Signal. 2015 Dec 1;27(12): 2452-66. https://doi.org/10.1016/j.cellsig.2015.09.011.

40. Veikkolainen V, Ali N, Doroszko M, Kiviniemi A, Miinalainen I, Ohlsson C, et al. Erbb4 regulates the oocyte microenvironment during folliculogenesis. Hum Mol Genet. 2020 Oct 10;29(17):2813-30. https://doi.org/10.1093/hmg/ddaa161.

41. Fattash I, Rooke R, Wong A, Hui C, Luu T, Bhardwaj P, et al. Miniature inverted-repeat transposable elements: discovery, distribution, and activity. Genome. 2013 Sep;56(9):475-86. https://doi.org/10.1139/gen-2012-0174.
42. Quintana-Murci L, Fellous M. The human Y chromosome: the biological role of a "functional wasteland." J Biomed Biotechnol 2001;1(1):18-24, The Human Y Chromosome: The Biological Role of a "Functional Wasteland", doi: https://doi.org/10.1155/S1110724301000080.

43. Tanabe H, Müller S, Neusser M, von Hase J, Calcagno E, Cremer M, et al. Evolutionary conservation of chromosome territory arrangements in cell nuclei from higher primates. Proc Natl Acad Sci U S A. 2002 Apr 2;99(7): 4424-9. https://doi.org/10.1073/pnas.072618599.

44. Bell J, Larson M, Kutzler M, Bionaz M, Löhr CV, Hendrix D. miRWoods: Enhanced precursor detection and stacked random forests for the sensitive detection of microRNAs. PLOS Computational Biology. 2019 Sep 10;15(10):e1007309.

45. Salas-Huetos A, James ER, Aston KI, Carrell DT, Jenkins TG, Yeste M. The role of miRNAs in male human reproduction: a systematic review. Andrology. 2020 Jan 1;8(1):7-26. https://doi.org/10.1111/andr.12714.

46. Salas-Huetos A, James ER, Aston Kl, Jenkins TG, Carrell DT, Yeste M. The Expression of miRNAs in human ovaries, oocytes, extracellular vesicles, and early embryos: a systematic review. Cells. 2019 Dec;4(12):8(12). https://doi. org/10.3390/cells8121564.

47. Sacchi S, Sena P, Degli Esposti C, Lui J, La Marca A. Evidence for expression and functionality of FSH and LH/hCG receptors in human endometrium. J Assist Reprod Genet. 2018 Sep;35(9):1703-12. https://doi.org/10.1007/s1081 5-018-1248-8.

48. Jima DD, Zhang J, Jacobs C, Richards KL, Dunphy CH, Choi WWL, et al. Deep sequencing of the small RNA transcriptome of normal and malignant human B cells identifies hundreds of novel microRNAs. Blood. 2010 Dec 2; 116(23):e118-27. https://doi.org/10.1182/blood-2010-05-285403.

49. Zhou Y, Wang Z, Chen X, Zhang J, Yang L, Liu S, et al. Identification of differentially expressed miRNAs and mRNAs in synovial of osteoarthritis via RNA-sequencing. BMC Med Genet [Internet]. 2020 Mar 2 [cited 2020 Dec 22]; 21. Available from: https://www.ncbi.nlm.nih.gov/pmc/articles/PMC7053084/

50. Wu S, Huang S, Ding J, Zhao Y, Liang L, Liu T, et al. Multiple microRNAs modulate p21Cip1/Waf1 expression by directly targeting its $3^{\prime}$ untranslated region. Oncogene. 2010 Apr 15;29(15):2302-8. https://doi.org/10.1038/onc.2 010.34 .

51. Sticht C, De La Torre C, Parveen A, Gretz N. miRWalk: An online resource for prediction of microRNA binding sites. PLoS One. 2018;13(10):e0206239.

52. Jiang C, Diao F, Sang Y-J, Xu N, Zhu R-L, Wang X-X, et al. GGPP-Mediated Protein Geranylgeranylation in Oocyte Is Essential for the Establishment of Oocyte-Granulosa Cell Communication and Primary-Secondary Follicle Transition in Mouse Ovary. PLoS Genet [Internet]. 2017 Jan 10 [cited 2021 Jan 28];13(1). Available from: https://www.ncbi.nlm.nih.gov/pmc/articles/ PMC5224981/

53. Lavoie $\mathrm{H}$, Therrien M. Regulation of RAF protein kinases in ERK signalling. Nat Rev Mol Cell Biol. 2015 May;16(5):281-98. https://doi. org/10.1038/nrm3979.

54. Butler T, Paul J, Europe-Finner N, Smith R, Chan E-C. Role of serine-threonine phosphoprotein phosphatases in smooth muscle contractility. Am J Phys Cell Phys. 2013 Jan 16:304(6):C485-504. https://doi.org/10.1152/ajpcell.001 61.2012.

55. Dong K, Zhang M-X, Liu Y, Su X-L, Chen B, Zhang X-L. Peroxisome proliferator-activated receptor alpha Expression changes in human pregnant myometrium. Reprod Sci. 2013 Jun;20(6):654-60. https://doi.org/1 $0.1177 / 1933719112461187$

56. Théry C, Witwer KW, Aikawa E, Alcaraz MJ, Anderson JD, Andriantsitohaina R, et al. Minimal information for studies of extracellular vesicles 2018 (MISEV2018): a position statement of the International Society for Extracellular Vesicles and update of the MISEV2014 guidelines. J Extracell Vesicles. 2018;7(1):1535750. https://doi.org/10.1080/20013078.2018.1535750.

57. Cakmak Genc G, Dursun A, Karakas Celik S, Calik M, Kokturk F, Piskin IE. IL28B, IL29 and micro-RNA 548 in subacute sclerosing panencephalitis as a rare disease. Gene. 2018 Dec 15;678:73-8. https://doi.org/10.1016/j.gene.201 8.07.062.

58. Habieb A, Matboli M, El-Tayeb H, El-Asmar F. Potential role of IncRNA-TSIX, miR-548-a-3p, and SOGA1 mRNA in the diagnosis of hepatocellular carcinoma. Mol Biol Rep. 2019 Aug;46(4):4581-90. https://doi.org/10.1007/ s11033-019-04810-x.

59. Weber JA, Baxter DH, Zhang S, Huang DY, Huang KH, Lee MJ, et al. The MicroRNA Spectrum in 12 body fluids. Clin Chem. 2010 Nov;56(11):1733-41. https://doi.org/10.1373/clinchem.2010.147405.

60. Home - Gene - NCBI [Internet]. [cited 2021 Jan 8]. Available from: https:// www.ncbi.nlm.nih.gov/gene/?term=. 
61. Yates AD, Achuthan P, Akanni W, Allen J, Allen J, Alvarez-Jarreta J, et al. Ensembl 2020. Nucleic Acids Res. 2020 Jan 8;48(D1):D682-8. https://doi. org/10.1093/nar/gkz966.

62. Waterhouse AM, Procter JB, Martin DMA, Clamp M, Barton GJ. Jalview version 2-a multiple sequence alignment editor and analysis workbench. Bioinformatics. 2009 May 1;25(9):1189-91. https://doi.org/10.1093/bioinforma tics/btp033.

63. Sievers F, Higgins DG. Clustal omega. Curr Protoc Bioinformatics. 2014 Dec 12;48(1):3.13.1-16. https://doi.org/10.1002/0471250953.bi0313s48.

64. Saitou N, Nei M. The neighbor-joining method: a new method for reconstructing phylogenetic trees. Mol Biol Evol. 1987 Jul 1;4(4):406-25. https://doi.org/10.1093/oxfordjournals.molbev.a040454.

65. Bolger AM, Lohse M, Usadel B. Trimmomatic: a flexible trimmer for Illumina sequence data. Bioinformatics. 2014 Aug 1;30(15):2114-20. https://doi.org/1 0.1093/bioinformatics/btu170

66. Friedländer MR, Mackowiak SD, Li N, Chen W, Rajewsky N. miRDeep2 accurately identifies known and hundreds of novel microRNA genes in seven animal clades. Nucleic Acids Res. 2012 Jan;40(1):37-52. https://doi. org/10.1093/nar/gkr688.

67. Robinson MD, McCarthy DJ, Smyth GK. edgeR: a Bioconductor package for differential expression analysis of digital gene expression data. Bioinformatics. 2010 Jan 1;26(1):139-40. https://doi.org/10.1093/bioinforma tics/btp616.

68. Metsalu T, Vilo J. ClustVis: a web tool for visualizing clustering of multivariate data using principal component analysis and heatmap. Nucleic Acids Res. 2015 Jul 1;43(W1):W566-70. https://doi.org/10.1093/nar/gkv468.

\section{Publisher's Note}

Springer Nature remains neutral with regard to jurisdictional claims in published maps and institutional affiliations.

Ready to submit your research? Choose BMC and benefit from:

- fast, convenient online submission

- thorough peer review by experienced researchers in your field

- rapid publication on acceptance

- support for research data, including large and complex data types

- gold Open Access which fosters wider collaboration and increased citations

- maximum visibility for your research: over $100 \mathrm{M}$ website views per year

At $\mathrm{BMC}$, research is always in progress.

Learn more biomedcentral.com/submissions 